UVX 2010 (2011) 3-9

DOI: $10.1051 / \mathrm{uvx} / 2011001$

(C) Owned by the authors, published by EDP Sciences, 2011

\title{
Impression par laser UV picoseconde pour la micro-électronique organique
}

\author{
L. Rapp, A.P. Alloncle, J. Ailuno et Ph. Delaporte \\ Laboratoire Lasers, Plasmas et Procédés Photoniques (LP3), UMR 6182 CNRS - Université \\ de la Méditerranée, C. 917, 13288 Marseille Cedex 9, France
}

\begin{abstract}
Résumé. La potentialité d'une technique d'écriture directe assistée par laser est démontrée dans le cadre de la fabrication d'un composant électronique de base, le transistor à effet de champ. L'impression de pixels à partir de divers matériaux susceptibles d'intervenir dans la fabrication d'un OFET (conducteur, semi-conducteur, diélectrique...) qu'ils soient organiques ou non, a été réalisée sous l'irradiation à $355 \mathrm{~nm}$ d'un laser picoseconde. L'éjection et le dépôt de la matière ont été étudiés comparativement au type de matériau utilisé: métal, polymère ou oligomère sous formes solides, encre à nanoparticules métalliques sous forme liquide. Des informations sur la morphologie des pixels obtenus, sur leur résolution spatiale, sur leur épaisseur ainsi que sur leurs propriétés d'adhésion ont été déduites d'analyses par microscopie optique, électronique à balayage (MEB) et/ou à force atomique (AFM). L'utilisation d'une couche intermédiaire servant à piéger le rayonnement incident et donc à protéger le matériau à imprimer a également été abordée.
\end{abstract}

\section{INTRODUCTION}

Les travaux de recherche, conduits dans les années 1970-1980 par les américains Heeger, Mac Diarmid et Shirakawa, sur propriétés conductrices des polymères, ont donné lieu en 2000 à l'attribution du prix Nobel de Chimie pour ces scientifiques et ont ouvert la voie à l'électronique organique. L'électronique devient légère, souple, s'adapte à des supports divers (tissus, films plastiques, papier ... ). Bien que loin des cadences de l'électronique basée sur le Silicium, ses applications sont multiples et elle représente un marché actuellement en plein essor. On peut citer à titre d'exemple, les écrans flexibles ou encore les capteurs divers et variés destinés à des applications de traçabilité, de sécurité ou d'analyse médicale...

Les techniques de fabrication de ces composés électroniques se sont donc développées sur les bases de productions de masse à bas coût répondant à des critères de fiabilité et de reproductibilité (flexographie, offset ...). Toutefois une modification du motif imprimé reste une opération lourde et coûteuse. Une technique d'écriture directe telle que le jet d'encre autorise une commande et un contrôle assistés par ordinateur qui lui confère donc une grande souplesse. Le point commun de tous ces procédés de fabrication, c'est qu'ils utilisent des matériaux solubles comme matière première pour la fabrication des composants électroniques. Hors, de nombreux matériaux aux propriétés électriques fort intéressantes ne possèdent pas de propriétés de solubilité, tels que, par exemple, les semi-conducteurs à petites molécules. En conséquence, une technique d'impression directe adaptable à tous types de matériaux (solubles ou non) serait d'un grand intérêt dans le cadre de ses applications.

Notre objectif est d'étudier le potentiel d'une technique laser très simple permettant d'imprimer des matériaux organiques sous forme solide ou liquide. Dans ce cadre, nous nous sommes attachés à la fabrication d'un élément de base commun a toutes les applications: le transistor organique à effet de champ (OFET).

La technique étudiée nécessite l'utilisation de deux substrats en contact l'un de l'autre ou maintenus parallèles à une distance très faible (dizaines de microns). L'un de ces substrat est recouvert d'une ou plusieurs couches minces de matériau (appelé donneur), et sous l'irradiation d'un laser impulsionnel, on

This is an Open Access article distributed under the terms of the Creative Commons Attribution-Noncommercial License 3.0, which permits unrestricted use, distribution, and reproduction in any noncommercial medium, provided the original work is properly cited. 
transfère en une seule étape, une pastille dimension micrométrique d'un substrat à l'autre. Il s'agit d'un procédé connu sous le nom de Laser Induced Forward Transfer (LIFT) [1].

Ce procédé a été largement étudié ces dernières années et a démontré en particulier son intérêt pour imprimer des matériaux de natures aussi différentes que des nanotubes [2], des céramiques [3], ou des matériaux biologiques particulièrement fragiles (cellules [4], ADN [5]).

\section{DISPOSITIF EXPÉRIMENTAL}

Un transistor organique à effet de champ (OFET) est constitué d'un empilement de plusieurs éléments qui peuvent être agencés selon diverses configurations. Les électrodes source et drain que l'on retrouve sur ou sous le semi-conducteur en fonction de l'architecture considérée et dont la distance détermine la longueur du canal du transistor (Figure 1). Le diélectrique et la grille placés sur ou sous les éléments précédemment cités selon que la configuration du transistor soit en «bottom gate» ou «top gate» (Figure 1).

a)

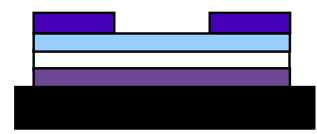

Semi-conducteur b)

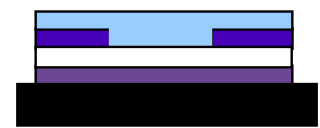

Source et Drain c)

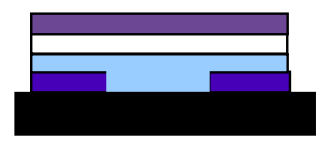

Isolant

Figure 1. Exemple de différentes configurations de transistors: a) «bottom gate» et «top contact» - b) «bottom gate » et «bottom contact »- c) « top gate » et «bottom contact».

L'étude de l'impression de pixels à partir de divers matériaux susceptibles d'intervenir dans la fabrication d'un OFET (conducteur, semi-conducteur, diélectrique) qu'ils soient organiques ou non a été réalisée.

L'objectif est d'obtenir une bonne résolution spatiale du pixel. En effet les électrodes sources et drain par exemple, définissent de par leur position et leur forme la longueur du canal du transistor. Cette dimension est directement reliée à la vitesse de commutation du transistor. L'objectif est de réaliser un canal le mieux défini possible et dont la longueur serait la plus petite possible. Par ailleurs le matériau ne doit pas être endommagé pendant le transfert et doit conserver ses propriétés électriques.

Dans cette optique, les expériences de LIFT ont été réalisée à l'aide d'un laser Nd:YAG (Continuum) de durée d'impulsion 50 ps. En raison des propriétés d'absorption de certains des matériaux utilisés la longueur choisie est de $355 \mathrm{~nm}$ (3ème harmonique). La forme et la dimension du pixel à imprimer est définie par l'association d'un masque et d'une lentille convergente permettant de prélever une partie $\mathrm{du}$ faisceau et d'en faire l'image sur le donneur. Les dimensions des pixels imprimés peuvent varier de $100 \times 100 \mu \mathrm{m}$ à $500 \times 500 \mu \mathrm{m}$. La position de l'échantillon est obtenue à l'aide de tables à translation de précision micrométrique $(\mathrm{x}, \mathrm{y}, \mathrm{z})$ et est contrôlée en visualisant le dépôt à l'aide d'une camera $\mathrm{CCD}$. Le substrat receveur est maintenu au contact du substrat donneur.

Des informations sur la morphologie des pixels obtenus, sur leur résolution spatiale, sur leur épaisseur ainsi que sur leurs propriétés d'adhésion ont été déduites d'analyses par microscopie optique, électronique à balayage (MEB) et/ou à force atomique (AFM).

\section{IMPRESSION DES PIXELS À PARTIR D’UN FILM MÉTALLIQUE}

\subsection{Film donneur solide}

Le film donneur est préparé par évaporation thermique sous vide d'Aluminium. Les pixels sont réalisés a partie de film de $100 \mathrm{~nm}$ d'épaisseur sur un substrat receveur de Silicium recouvert d'une couche d'oxyde de Silicium. Des dépôts bien résolus sont obtenus à partir de $0,46 \mathrm{~J} / \mathrm{cm}^{2}$ (figure 2). 


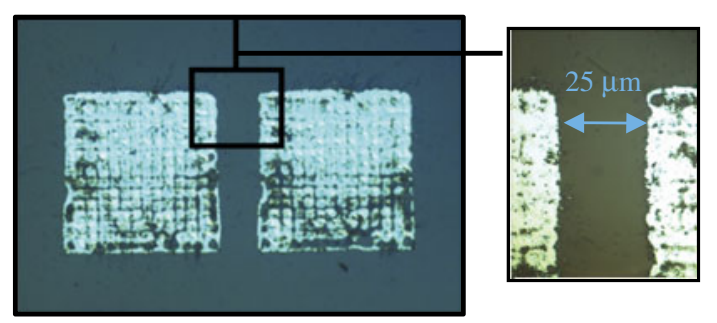

Figure 2. Impression de pixels d'Aluminium $100 \mu \mathrm{mx} 100 \mu \mathrm{m}$ à partir d'un film solide de $100 \mathrm{~nm}$ d'épaisseur; $\mathrm{F}=0,46 \mathrm{~J} / \mathrm{cm}^{2}$.

Toutefois on constate une grande dépendance du profil d'énergie du faisceau dans l'homogénéité du pixel déposé. L'augmentation de la fluence provoque une destruction du dépôt formé, voire un endommagement du substrat receveur et la multiplication de débris autour du pixel obtenu. La conductivité du matériau déposé est très proche de celle du matériau initial. L'impression de ce type de pixel par LIFT est à priori tout à fait adaptée à leur utilisation comme électrodes sources/drain. La bonne résolution spatiale obtenue et le peu de débris résultants de l'ablation du donneur, peuvent permettre d'envisager la formation d'un canal d'une longueur dont l'ordre de grandeur serait la dizaine de microns. Toutefois, la fluence étant donné la fluence élevée nécessaire à la génération d'un pixel, une autre solution est également étudiée.

\subsection{Film donneur liquide}

Le film donneur est alors réalisé par spin coating (2900rpm pendant 20s) d'une encre contenant des nanoparticules d'argent (CABOT). Le substrat receveur $\left(\mathrm{Si}-\mathrm{SiO}_{2}\right)$ est maintenu à une distance de $50 \mathrm{~mm}$ du donneur. Le faisceau laser est dans ce cas focalisé sur la couche liquide qui est absorbante au rayonnement. A une fluence de $0,001 \mathrm{~J} / \mathrm{cm}^{2}$, une gouttelette d'encre est transférée sur le substrat receveur. Après recuit de cette encre à $150^{\circ} \mathrm{C}$, ces dépôts sont observés au microscope optique et caractérisés à l'AFM (figure 3). Il en résulte un pixel circulaire d'un diamètre de $20 \mu \mathrm{m}$ et d'une épaisseur de $250 \mathrm{~nm}$. Aucun débris n'est visible autour du dépôt. Le mécanisme de génération de ces gouttelettes a été récemment étudié en détail par Duocastella [6]. Il a montré qu'une bulle de vapeur était générée dans le film liquide lors de l'interaction laser/matière. Cette bulle initialement à haute pression et haute température suit un mouvement de détente, sa dimension augmente. Puis, dans des conditions optimales concernant l'énergie déposée et l'épaisseur du film liquide, elle s'effondre. Un jet liquide extrêmement fin est alors formé et dirigé perpendiculairement au substrat donneur. Ce jet transporte le liquide nécessaire à la création d'une gouttelette sur le substrat receveur.

Des lignes sont formées en juxtaposant des gouttelettes avec un très faible recouvrement (figure 3 ). Les mesures de résistivité de ces lignes nous donnent des valeurs comprises entre 5 et $10 \mu \Omega$.cm.

a)

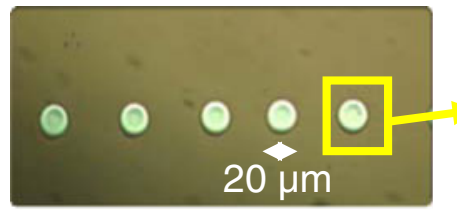

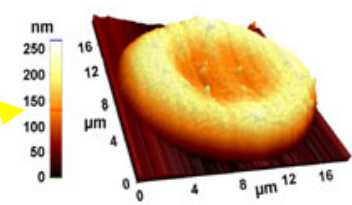

b)

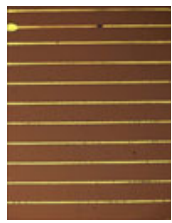

Figure 3. Impression de pixels à partir de gouttelettes d'encre contenant des nanoparticules d'Argent sur un substrat de Silicium recouvert d'une couche d'oxyde. a) impression successive de gouttelettes de $20 \mu \mathrm{m}$ de diamètre à une fluence de $0,001 \mathrm{~J} / \mathrm{cm}^{2}$, (recuit $150^{\circ} \mathrm{C}$. b) impression de lignes par juxtaposition de gouttelettes (léger recouvrement). 
Il est possible de diminuer cette valeur en augmentant la température du recuit. Elle doit toutefois rester faible si on souhaite que ce procédé soit compatible avec l'utilisation de substrats ne supportant pas des températures élevées (polyéthylène téréphtalate PET...).

\section{IMPRESSION DE PIXELS À PARTIR D'UN FILM ORGANIQUE}

\subsection{Film donneur à base de polymère}

Dans le cadre d'application aux transistors organiques, des polymères peuvent être utilisés pour différentes fonctions. Le polyméthyle méthacrylate (PMMA) a une constante diélectrique compatible avec une utilisation comme couche isolantes d'un transistor (e = 2,6 à $1 \mathrm{MHz}, \mathrm{e}=3,9$ à $60 \mathrm{MHz}$ ). Il a par ailleurs une bonne résistance mécanique et et une faible température de polymérisation (inférieure à $160^{\circ} \mathrm{C}$ ) ce qui le rend compatible avec l'utilisation de substrat basse température. Le poly(3,4-ethylenedioxythiophene)-poly(styrenesulfonate) PEDOT:PSS est un polymère conducteur commercialisé par H.C. Starck. Son travail de sortie à hauteur de 5,2 eV en fait un bon candidat pour la réalisation des électrodes source/drain [7]. Par ailleurs il existe beaucoup d' autres matériaux susceptibles d'être utilisés pour ce genre d'application. Par exemple, des polymères aux propriétés semi-conductrices (Poly(3-hexylthiophène) régiorégulier ou P3HT ou le Poly(3,3"'-didodécylquarterthiophène) ou PQT12) ont également été utilisés pour l'impression de transistors avec une technologie de jet d'encre $[7,8]$.

a)

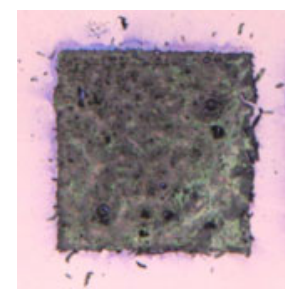

b)

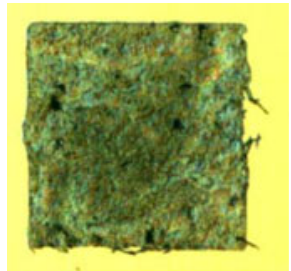

c)

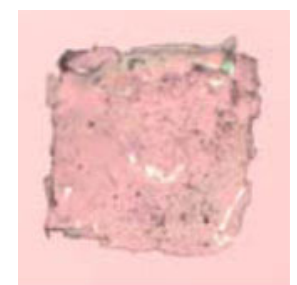

Figure 4. Impression de pixels de $500 \mu \mathrm{mx} 500 \mu \mathrm{m}$ à partir films donneurs polymères: a) PEDOT:PSS; épaisseur du donneur $300 \mathrm{~nm} ; \mathrm{F}=0,4 \mathrm{~J} / \mathrm{cm}^{2}$; b) PEDOT:PSS imprimé par LIFT avec une couche absorbante intermédiaire de $25 \mathrm{~nm}$ d'or; épaisseur du donneur $300 \mathrm{~nm} ; \mathrm{F}=0,2 \mathrm{~J} / \mathrm{cm}^{2} ; \mathrm{c}$ ) PMMA; épaisseur du donneur $700 \mathrm{~nm} ; \mathrm{F}=0,2 \mathrm{~J} / \mathrm{cm}^{2}$.

L'impression de pixels par LIFT sur un substrat de Silicium recouvert d'une couche de d'oxyde de Silicium de deux types de polymères, le PEDOT:PSS et le PMMA, a été étudiée pour diverses fluences. Les donneur de PMMA et de PEDOT:PSS sont réalisés par spin coating (respectivement $1000 \mathrm{rpm}$ et $2000 \mathrm{rpm}$ pendant $20 \mathrm{~s}$ ) sur des substrat de quartz suprasil (Hellma) puis le solvant est évaporé pendant 20 minutes au four, respectivement à $80^{\circ} \mathrm{C}$ pour l'un et $120^{\circ} \mathrm{C}$ pour l'autre. Les images des pixels obtenus pour les conditions optimales sont montrés (Figure 4(a) et 4(c)). Le pixel de PEDOT:PSS est très bien défini, mais de nombreux débris sont visibles sur et en dehors de la zone déposée. Le pixel de PMMA est aussi bien défini mais semble découpé moins précisément. Ces deux matériaux sont très transparents à la longueur d'onde du laser (70\% de transmission pour le PEDOT:PSS et presque $100 \%$ pour le PMMA). La forte intensité lumineuse (l'intensité lumineuse moyenne sur la zone irradiée est largement supérieure à $10^{9} \mathrm{~W} / \mathrm{cm}^{2}$.) associée à la présence de défauts ou d'impuretés dans les couches de polymères provoque une absorption du rayonnement qui permet un dépôt d'énergie dans un volume de matériau suffisant pour initier une rupture des films sous l'action de contraintes mécaniques. Toutefois les épaisseurs des pixels déposés sont beaucoup faible que les épaisseurs initiales (400 nm d'épaisseur déposée pour le PMMA pour $700 \mathrm{~nm}$ d'épaisseur initiale de la couche). La dynamique d'éjection de ces couches à été largement décrite dans des travaux précédemment publiés [9]. Il a été montré entre l'optimisation du procédé en contrôlant le dépôt d'énergie du laser par l'insertion d'une couche très absorbante entre le substrat et le film à imprimer [10]. La figure 4(b) illustre cette optimisation. La 
couche absorbante dans ce cas précis est composée de $25 \mathrm{~nm}$ d'or. Le pixel de PEDOT:PSS est imprimé à une fluence plus faible et les débris ont disparu. Des analyses en microscopie électronique montrent que des gouttelettes d'or de dimension nanométrique subsistent à la superficie du pixel. Des mesures des propriétés électriques sont effectuées sur des lignes de PEDOT:PSS constituées de pixels juxtaposés avec un léger recouvrement. Il en résulte que la résistivité moyenne du PEDOT:PSS est estimée à $5 \times 10^{-3} \Omega$.cm; ce qui est proche de celle mesurée pour du PEDOT:PSS imprimé par jet d'encre $[7,8]$. Par contre l'adhésion des pixels (PMMA et PEDOT:PSS) sur le substrat de $\mathrm{Si} / \mathrm{SiO}_{2}$ est assez faible. C'est un point qu'il sera nécessaire de contrôler pour l'avenir de ce procédé.

\subsection{Film donneur à base d'oligomère}

Contrairement aux polymères, les oligomères sont composés de petites molécules. Ces composés ne sont généralement pas solubles et des couches minces de matériau sont obtenus par évaporation sous vide. Ces films ont généralement des structures bien ordonnées ce qui leur confère des propriétés électriques remarquables. Par contre, contrairement aux films polymères, ils ont une résistance mécanique très faible. Dans ce type de matériaux on retrouve par exemple la phthalocyanine de cuivre $(\mathrm{CuPc})$ et l'hexadecafluorophthalocyanine de cuivre $\left(\mathrm{F}_{16} \mathrm{CuPc}\right)$ qui sont des semi-conducteurs respectivement de type $\mathrm{p}$ et $\mathrm{n}$. Ce sont des composés stables à l'air et qui possèdent des mobilités qui peuvent aller jusqu'à $4.10^{-2} \mathrm{~cm}^{2} \mathrm{~V}^{-1} \mathrm{~s}^{-1}$ pour le $\mathrm{CuPc}$ et $3.10^{-2} \mathrm{~cm}^{2} \mathrm{~V}^{-1} \mathrm{~s}^{-1}$ pour le $\mathrm{F}_{16} \mathrm{CuPc}$. Par ailleurs, le spectre d'absorption de ces composés montre une bande d'absorption autour de la longueur d'onde de $355 \mathrm{~nm}$. L'impression de pixels de CuPC et de $\left(\mathrm{F}_{16} \mathrm{CuPc}\right)$ par LIFT sur un substrat de $\mathrm{Si} / \mathrm{SiO}_{2}$ a été étudiée pour diverses fluences. Les pixels obtenus pour des conditions optimales sont montrés figure 5. Les pixels sont homogènes et bien résolus spatialement malgré quelques éclaboussures visibles à leur périphérie. L'impression des pixels s'effectue dans un domaine très étroit de fluence. En effet une fluence de $0,2 \mathrm{~J} / \mathrm{cm}^{2}$ entraine déjà une détérioration du matériau déposé.

a)

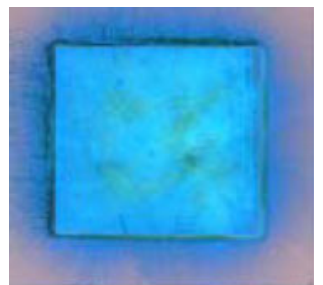

b)

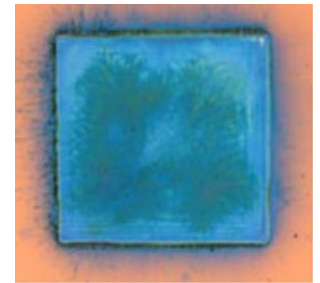

Figure 5. Impression de pixels de $500 \mu \mathrm{mx} 500 \mu \mathrm{m}$ à partir films donneurs oligomères: a) CuPc; épaisseur du donneur $100 \mathrm{~nm} ; \mathrm{F}=0,1, \mathrm{~J} / \mathrm{cm}^{2}$; b) F16CuPc épaisseur du donneur $100 \mathrm{~nm} ; \mathrm{F}=0,1 \mathrm{~J} / \mathrm{cm}^{2}$.

Par contre, le dépôt de ces semi-conducteurs est compatible avec l'utilisation de substrats flexibles basse température. La figure 5(a)) montre l'impression par LIFT de lignes de CuPc (500 $\mu$ m de large) et de lignes d'encre contenant des nanoparticules d'argent sur des substrats composés de $50 \mu \mathrm{m}$ de PET recouvert de $60 \mathrm{~nm}$ d'aluminium (grille) et enfin de $4 \mathrm{~nm}$ de $\mathrm{SiO}_{2}$ (diélectrique). Elles sont imprimée par LIFT selon une configuration transistor en top contact. Aucun endommagement du substrat n'apparaît après le dépôt. Des tests de torsions et flexions simples sont effectués sur l'échantillon. Aucun dommage n'est observé par microscopie à balayage dans la structure des dépôts, que se soit le semi-conducteur ou l'encre à nanoparticules.

\section{CONCLUSION - RÉALISATION DE TRANSISTORS}

Pour les essais transistors, les substrats choisis sont composés de Silicium recouvert d'une couche d'oxyde de Silicium de $300 \mathrm{~nm}$ d'épaisseur (Vegatec). Des transistors composés de CuPc imprimé 
par LIFT et d'encre à nanoparticules d'argent (figure 6(b)) ont été réalisés en configuration top et bottom contact. D'autres ont été réalisés à partir de $\mathrm{F}_{16} \mathrm{CuPc}$ et d'électrodes d'aluminium (figure 6(c)) en configuration bottom contact. Ces études font l'objet des références [11] et [12]. Les résultats des caractérisations électriques montrent l'établissement d'un effet de champ, donc un transistor fonctionnel, dans tous les cas. Toutefois les performances en termes de mobilité restent encore inférieures (très légèrement pour certaines configurations) au même transistor obtenu par évaporation sous-vide du semi-conducteur et des électrodes. Des études sont actuellement en cours afin d'optimiser l'injection et le transport des charges dans le transistor, notamment en mettant en oeuvre un dépôt par LIFT de plusieurs couches à la fois.
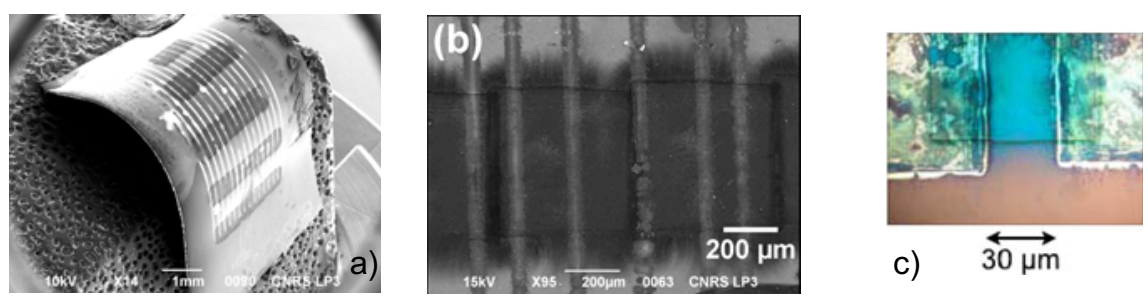

Figure 6. a) Impression de lignes de CuPC de $500 \mu \mathrm{m}$ de large et d'encre à nanoparticules d'argent sur un substrat flexible; b) transistor réalisé en bottom contact par LIFT d'encre à nanoparticules d'argent et de CuPc sur substrat $\mathrm{Si} / \mathrm{SiO}_{2} ;$ c)transistor réalisé en bottom contact par LIFT d'aluminium et de $\mathrm{F}_{16} \mathrm{CuPc}$ sur substrat $\mathrm{Si} / \mathrm{SiO}_{2}$.

Le dépôt par LIFT de l'isolant de grille à également été étudié. Sur un transistor ou le semiconducteur $(\mathrm{CuPc})$ et les électrodes source/drain (or) sont formés par évaporation thermique sous vide, un pixel de PMMA est imprimé par LIFT, la grille (Al) est ensuite évaporée sous vide en configuration top gate [13]. Les caractérisations électriques montrent un transistor tout à fait opérationnel et démontrent la capacité de déposer le PMMA sans endommager les éléments préalablement formés.

La technique du LIFT peut donc être utilisée pour imprimer des matériaux de natures et de propriétés très diverses. Elle possède un potentiel certain dans le cadre d'applications en électronique organique.

\section{Remerciements}

Les auteurs remercient S. Nénon, A.K. Diallo, CH Videlot Ackerman et F. Fages du CiNAM (Marseille) pour leur collaboration dans ces études.

\section{Références}

[1] Bohandy J., Kim B.F., Adrian F.J., Jette A.N., J. Appl. Phys. 63 (4) (1988) 1158

[2] Boutopoulos C., Pandis C., Giannakopoulos K., Pissis P., Zergioti I., Appl. Phys. Lett. 96 (2010) 041104

[3] Kaur K.S., Fardel R., May-Smith T.C., Nagel M., Banks D.P., Grivas C., Lippert T., Eason R.W., J. Appl. Phy. 105 (2009) 113119

[4] Serra P., Colina M., Fernandez-Pradas J.M., Sevilla L., Morenza J.L., Appl. Phys. Lett. 85 (9) (2004) 1639

[5] Hopp B., Smausz T., Kresz N., Barna N., Bor Z., Kolozvari L., Chrisey D.B., Szabo A., Nogradi A., Tissue Eng. 111817 (2005)

[6] Duocastella M., Fernandez-Pradas J.M., Serra, P., Morenza J.L., Appl. Phys. A. 93 (9) (2008) 453-456

[7] Barret M., Sanaur S, Collot P., Organic Electronics, 9 (2008) 1093-1100

[8] Barret M., Sanaur S, Collot P., Mater. Res. Soc. Symp. Proc., (2007) 1003 
[9] Rapp L., Cibert Ch., Alloncle A.P., Delaporte Ph., Nénon S., Videlot-Ackerman C., Fages F., SPIE 7131L, (2009)

[10] Rapp L., Cibert Ch., Alloncle A.P., Delaporte Ph., Applied Surface Sciences, 255 (10) (2009) 5439-5443

[11] Rapp L., Diallo A.K., Alloncle A.P., Videlot-Ackerman C., Fages F., Delaporte Ph., Appl. Phys. Lett. 95, (2009) 171109

[12] Rapp L., Alloncle A.P., Diallo A.K, Delaporte Ph, Nénon S., Videlot-Ackerman C., Fages F., Proceeding of MRS Fall meeting (2009) Boston

[13] Rapp L., Nénon S., Diallo A.K, Alloncle A.P., Delaporte Ph, Videlot-Ackerman C., Fages F., Mater. Res. Soc. Symp. Proc. 1270 (2010) 11-10 